Pacific Journal of Mathematics

ORTHOGONAL POLYNOMIALS ASSOCIATED WITH THE 


\title{
ORTHOGONAL POLYNOMIALS ASSOCIATED WITH THE ROGERS-RAMANUJAN CONTINUED FRACTION
}

\author{
Waleed A. Al-Salam and Mourad E. H. Ismail
}

\begin{abstract}
We characterize the symmetric orthogonal polynomials $\left\{P_{n}(x)\right\}$ such that $\left\{P_{n}\left(q^{n} x\right)\right\}$ is also orthogonal. This leads to orthogonal polynomials related to the denominator polynomials of the continued fractions of Rogers, Ramanujan, and Carlitz. We establish the orthogonality relation for these polynomials and show that the function $\Sigma_{0}^{\infty} q^{n^{2}} z^{n} /(q ; q)_{n}$ that appear in the aforementioned continued fractions have only real and simple zeros.
\end{abstract}

1. Introduction. In recent years we have seen tremendous and renewed interest in orthogonal $q$-polynomials both new and old. Among these are the $q$-Krawtchouk, the $q$-Meixner and the $q$-Laguerre (or what Chihara [4] calls generalized Stieltjes-Wigert polynomials). These sets are all of the form $\left\{F_{n}\left(q^{n} x\right)\right\}$, they are all orthogonal although the polynomial sets $\left\{F_{n}(x)\right\}$ are not themselves orthogonal.

It is therefore natural to ask if there are other orthogonal polynomial sets (OPS) $\left\{P_{n}(x)\right\}$ such that the corresponding set $\left\{P_{n}\left(q^{n} x\right)\right\}$ is also an OPS.

We consider this problem in $\$ 2$ and give a complete answer in the symmetric case obtaining a $q$-analog of the Tchebicheff polynomials (see formula (2.1)) and the polynomials studied by Geronimus [5].

The methods we use to obtain the measure with respect to which these polynomials are orthogonal work for a much more general class of polynomials. We thus introduce in $\$ 3$ the polynomial set $\left\{U_{n}(x, a, b)\right\}$ defined by means of (3.1). These polynomials include as special case not only the $q$-Tchebicheff (2.1) but also the $q$-Lommel [7]. We obtain for this OPS a generating and explicit representation. In $\S 4$ we construct the measure with respect to which these are orthogonal. Finally in $\$ 5$ we discuss briefly some related continued fractions of Rogers, Ramanujan, and Carlitz. Our polynomials $\left\{U_{n}(x ; a, b)\right\}$ are generated by

$$
\begin{gathered}
U_{0}(x ; a, b)=1, \quad U_{1}(x ; a, b)=x(1+a), \\
U_{n+1}(x ; a, b)=x\left(1+a q^{n}\right) U_{n}(x ; a, b)-b q^{n-1} U_{n-1}(x ; a, b), \quad n>0 .
\end{gathered}
$$

The most general symmetric polynomial solution to the above recurrence is also investigated in $\$ 5$. Observe that $U_{n}(x ; a, b)$ is a polynomial of degree $n$ in both $x$ and $a$. We discuss only the orthogonality of $U_{n}(x ; a, b)$ as functions of $x$ for fixed $a, a>-1$. As functions of $a$ the $U_{n}$ 's are 
orthogonal on an unbounded interval and the analysis becomes much more difficult. We hope to investigate this in a future work together with Turan inequalities.

2. A characterization theorem. In this section we look for all symmetric orthogonal polynomial sets (OPS) $\left\{P_{n}(x)\right\}$ that have the property that $\left\{P_{n}\left(q^{n} x\right)\right\}$ is also an OPS. We prove

THEOREM. A necessary and sufficient condition for a symmetric OPS $\left\{P_{n}(x)\right\}$ to be orthogonal and $\left\{P_{n}\left(q^{n} x\right)\right\}$ to be also an OPS is that $\left\{P_{n}(x)\right\}$ satisfies the recurrence

$$
\begin{aligned}
& P_{n}(x)=x P_{n-1}(x)-\lambda_{n} P_{n-2}(x) \quad(n \geq 2) \\
& P_{0}(x)=1, \quad P_{1}(x)=c x \quad(c \neq 0)
\end{aligned}
$$

where $\lambda_{2}$ is an arbitrary positive number and $\lambda_{n}=q^{2 n-4}$ for $n \geq 3$.

To prove this theorem we note first that because $\left\{P_{n}(x)\right\}$ were assumed symmetric then, without loss of generality, we may assume that they satisfy the recurrence

$$
\begin{aligned}
& P_{n}(x)=x P_{n-1}(x)-\lambda_{n} P_{n-2}(x) \quad(n \geq 2) \\
& P_{0}(x)=1 \quad \text { and } \quad P_{1}(x)=c x .
\end{aligned}
$$

If the polynomial set $Q_{n}(x)=P_{n}\left(q^{n} x\right)$ is to be also orthogonal then we must have

$$
P_{n}\left(q^{n} x\right)=q^{2 n-1} x P_{n-1}\left(q^{n-1} x\right)-\Lambda_{n} P_{n-2}\left(q^{n-2} x\right) \quad(n \geq 2)
$$

where $\Lambda_{n} \neq 0$ (as we shall see we need not assume that $\Lambda_{n}>0$ ) for $n=2,3,4, \ldots$.

Furthermore, symmetry implies that we can write

$$
P_{n}(x)=\sum_{k=0}^{n} p(n, n-2 k) x^{n-2 k}=p(n, n) x^{n}-\mu_{n} x^{n-2}+\cdots
$$

so that $p(n, n)=c$ and $\mu_{n}=\mu_{n-1}+c \lambda_{n}$. It follows easily from (2.2) and (2.3) that $P_{2 n}(0)=(-1)^{n} \lambda_{2} \lambda_{4} \cdots \lambda_{2 n}=(-1)^{n} \Lambda_{2} \Lambda_{4} \cdots \Lambda_{2 n}$ so that we have

$$
\lambda_{2 n}=\Lambda_{2 n} \quad(n \geq 1) .
$$


Now equating coefficients of $x^{n-2 k}$ in (2.2) and (2.3) we get, for $n \geq 2$, respectively

$$
\begin{gathered}
p(n, n-2 k)=p(n-1, n-1-2 k)-\lambda_{n} p(n-2, n-2 k) \\
P(n, n-2 k)= \\
q^{2 k} p(n-1, n-1-2 k) \\
-\Lambda_{n} q^{4 k-2 n} p(n-2, n-2 k)
\end{gathered}
$$

from which we obtain for $k=1,2, \ldots, n ; n \geq 1$

$$
\begin{aligned}
& p(2 n, 2 n-2 k)\left(1-q^{2 k}\right) \\
& \quad=q^{2 k} \lambda_{2 n}\left(1-q^{2 k-4 n}\right) p(2 n-2,2 n-2 k)
\end{aligned}
$$

(2.6) $p(2 n, 2 n-2 k)\left(1-q^{2 k}\right)$

$$
=\left(\lambda_{2 n+1}-q^{4 k-4 n-2} \Lambda_{2 n+1}\right) p(2 n-1,2 n+1-2 k)
$$

(2.7) $p(2 n+1,2 n+1-2 k)\left(1-q^{2 k}\right)$

$$
=q^{2 k}\left(\lambda_{2 n+1}-q^{2 k-4 n-2} \Lambda_{2 n+1}\right) p(2 n-1,2 n+1-2 k)
$$

and

$$
\begin{aligned}
& p(2 n-1,2 n-1-2 k)\left(1-q^{2 k}\right) \\
& \quad=\lambda_{2 n}\left(1-q^{4 k-4 n}\right) p(2 n-2,2 n-2 k) .
\end{aligned}
$$

Now iterating (2.5) we get

(2.9) $p(2 n, 2 n-2 k)=\varepsilon_{k, n} q^{k(k+1)} \frac{\left(q^{2 k-4 n}, q^{2}\right)}{\left(q^{2}, q^{2}\right)_{k}} \lambda_{2 n} \lambda_{2 n-2} \cdots \lambda_{2 n-2 k+2}$.

Similarly (2.8) yields

(2.10) $p(2 n-1,2 n-1-2 k)$

$$
=\varepsilon_{k, n} q^{k(k-1)} \frac{\left(q^{2 k-4 n+2} ; q^{2}\right)_{k}}{\left(q^{2} ; q^{2}\right)_{k}} \lambda_{2 n} \lambda_{2 n-2} \cdots \lambda_{2 n-2 k+2}
$$

where $\varepsilon_{k, n}=1$ if $k=n$ and $=c$ if $k<n$.

Solving for $\Lambda_{2 n+1}$ from (2.5) and (2.6) and also finding $\lambda_{2 n+1}$ from (2.7) and (2.8), in both cases with $k=1$, and then eliminating $\Lambda_{2 n+1}$ from the resulting equation we get for $n \geq 2$

(2.11) $\lambda_{2 n+1} q^{4 n}\left(1-q^{2}\right)+q^{4}\left(1-q^{4 n-2}\right) \lambda_{2 n}=\lambda_{2 n+2}\left(1-q^{4 n}\right)$. 
Now $k=1$ in (2.8) yields $-\mu_{2 n-1}\left(1-q^{2}\right)=\lambda_{2 n}\left(1-q^{4-4 n}\right) c$ so that

$$
\mu_{2 n}=c \lambda_{2 n} q^{4-4 n} \frac{1-q^{4 n-2}}{1-q^{2}} .
$$

The case $k=1$ of (2.6) and (2.12) gives

$$
\Lambda_{2 n+1}-q^{4 n-2} \lambda_{2 n+1}=\lambda_{2 n}\left(1-q^{4 n-2}\right) q^{2} \text {. }
$$

Next (2.6) with $k=n$ gives

$$
p(2 n, 0)\left(1-q^{2 n}\right)=\left(\lambda_{2 n+1}-q^{-2} \lambda_{2 n+1}\right) p(2 n-1, n) .
$$

But (2.9) and (2.10) imply

$$
\frac{p(2 n, 0)}{p(2 n-1,1)}=-\frac{\lambda_{2}}{c} q^{4(n-1)} \frac{1-q^{2}}{1-q^{2 n}} .
$$

Putting (2.15) in (2.14) and eliminating $\Lambda_{2 n+1}$ with (2.13) we get ( $\left.n \geq 2\right)$

$$
\left(1-q^{4 n-4}\right) \lambda_{2 n+1}=\lambda_{2 n}\left(1-q^{4 n-2}\right)-\frac{\lambda_{2}}{c} q^{4 n-4}\left(1-q^{2}\right) .
$$

Now (2.16) and (2.11) determine $\lambda_{n}$ completely if $\lambda_{3}, \lambda_{4}$ are given. In fact it is easy to see that (2.16) and (2.11) are satisfied by $c \lambda_{n}=\lambda_{2} q^{2 n-4}$ for $n \geq 4$.

We have from (2.12)

$$
c \lambda_{4}=\mu_{3} \frac{q^{4}}{1+q^{2}} \quad \text { and } \quad \mu_{4}=\mu_{3} \frac{1-q^{6}}{1-q^{4}} .
$$

If we put the computed expressions for $P_{3}(x), P_{4}(x)$ and $P_{5}(x)$ in (2.3) and equate coefficients of like powers of $x$, we get

$$
c \lambda_{5}=\frac{q^{4}}{1+q^{2}}\left(\lambda_{3}+\lambda_{4}\right) .
$$

Similarly we set $n=6$ in (2.3) and after some calculations we get

$$
c \lambda_{6}+\mu_{5} q^{10}=\mu_{6} q^{8}
$$

and

$$
\left(\lambda_{2} \lambda_{4}+\lambda_{5} \mu_{3}\right) q^{4}=\mu_{4} \lambda_{6} .
$$

Formulas (2.20) and (2.17) yield

$$
c \lambda_{6}=\frac{q^{8}}{1+q^{2}} \mu_{3} .
$$


On the other hand (2.19) and (2.21) give

$$
\mu_{5}=\mu_{3}\left(1+q^{4}\right) \text {. }
$$

But $\mu_{5}=\mu_{3}+c \lambda_{4}+c \lambda_{5}=\mu_{3}\left(1+q^{4}\right)$ implies $c \lambda_{4}+c \lambda_{5}=\mu_{3} q^{4}$ which together with (2.18) and (2.17) establish

$$
c \lambda_{3}=\mu_{3} \frac{q^{2}}{1+q^{2}}=\left(\lambda_{2}+c \lambda_{3}\right) \frac{q^{2}}{1+q^{2}} .
$$

Thus

$$
c \lambda_{3}=\lambda_{2} q^{2}, \quad c \lambda_{4}=\lambda_{2} q^{4} \text { and } c \lambda_{5}=\lambda_{2} q^{6} .
$$

Therefore we have proved that

$$
c \lambda_{n}=\lambda_{2} q^{2 n-4} \quad(n \geq 3)
$$

and $\lambda_{2}>0$ is arbitrary. This completes the proof of the characterization theorem.

It is easy to write the explicit expression for $P_{n}(x)$ and find a generating function. However we shall consider a more general class of polynomials in $\S 3$, when $c=1$, and in $\$ 5$ for general $c$, see (5.8). The only difference is that in the above calculations $b$ is replaced by $b q^{-2}$ then $q$ is replaced by $\sqrt{q}$ so the recurrence relation (2.1) becomes

$$
P_{n}(x)=x P_{n}(x)-b q^{n-1} P_{n-1}(x), n \geq 2, P_{0}(x)=1, P_{1}(x)=c s .
$$

3. The polynomials $U_{n}(x ; a, b)$. We define the sequence of polynomials $U_{n}(x ; a, b)$ recursively by

$$
\begin{aligned}
U_{n+1}(x ; a, b)= & x\left(1+a q^{n}\right) U_{n}(x ; a, b) \\
& -b q^{n-1} U_{n-1}(x ; a, b), \quad n>0,
\end{aligned}
$$

with $0<q<1,0<b,-1<a$ and

$$
U_{0}(x ; a, b)=1, \quad U_{1}(x ; a, b)=x(1+a) .
$$

The polynomials of $\S 2$ when $c=1$ correspond to the special case $a=0$ and $b=q$. The $q$-Lommel polynomials [7] are $U_{n}\left(2 / x ;-q^{\nu}, q^{\nu}\right)$. As $q \rightarrow 1$, $U_{n}(x ; a, b)$ reduces to the Tchebicheff polynomial of the second kind provided that $a$ does not tend to -1 .

The polynomials of the second kind $U_{n}^{*}(x ; a, b)$, see Askey and Ismail [2] or Pollaczek [9], satisfy (3.1) and the initial conditions

$$
U_{0}^{*}(x ; a, b)=0, \quad U_{1}^{*}(x ; a, b)=1+a .
$$


It is easy to see that

$$
U_{n}^{*}(x ; a, b)=(1+a) U_{n-1}(x ; q a, q b), \quad n>0 .
$$

THEOREM 3.1. The polynomials $\left\{U_{n}(x ; a, b)\right\}$ have the generating function

$$
\sum_{0}^{\infty} U_{n}(x ; a, b) t^{n}=\sum_{0}^{\infty} \frac{\left(\frac{b t}{x a} ; q\right)_{m}}{(x t ; q)_{m+1}}(x a t)^{m} q^{m(m-1) / 2} .
$$

Proof. Denote the left side of (3.5) by $U(x, t)$. Multiply (3.1) by $t^{n+1}$ and add the resulting equalities for $n=1,2, \ldots$ to get

$$
U(x, t)=\frac{1}{1-x t}+\frac{t(x a-b t)}{1-x t} U(x, q t),
$$

which, when iterated, leads to

$$
U(x, t)=\sum_{0}^{\infty} \frac{(x a t)^{n}}{(x t ; q)_{n+1}}\left(\frac{b t}{x a} ; q\right)_{n} q^{n(n-1) / 2} .
$$

One way to justify the above formal steps is to observe that the right side of (3.5) satisfies the $q$-difference equation (3.6) and is an analytic function of $t$ in a neighborhood of $t=0$. The coefficients of its Taylor series expansion about $t=0$ then will satisfy (3.1) and the initial conditions (3.2). This identifies the Taylor series coefficients as $U_{n}(x ; a, b)$ and the proof is complete.

Using the analogy with the $q$-Lommel polynomials [7] it is not difficult to guess that

$$
U_{n}(x ; a, b)=\sum_{k=0}^{[n / 2]} \frac{(-a ; q)_{n-k}(q ; q)_{n-k} x^{n-2 k}(-b)^{k}}{(-a ; q)_{k}(q ; q)_{k}(q ; q)_{n-2 k}} q^{k(k-1)}
$$

which can be proved by showing that the right side of (3.7) satisfies (3.1) and (3.2). Formula (3.7) can also be derived from the generating function (3.5) and the $q$-binomial Theorem, Slater [12, p. 92]

$$
\sum_{0}^{\infty} \frac{(\alpha ; q)_{n}}{(q ; q)_{n}} z^{n}=\frac{(\alpha z ; q)_{\infty}}{(z ; q)_{\infty}}
$$

as follows. Start with

$$
\frac{\left(\frac{b t}{x a} ; q\right)_{m}}{(x t ; q)_{m+1}}=\frac{\left(\frac{b t}{x a} ; q\right)_{\infty}}{\left(\frac{b t}{x a} q^{m} ; q\right)_{\infty}} \cdot \frac{\left(x t q^{m+1} ; q\right)_{\infty}}{(x t ; q)_{\infty}}
$$


apply the $q$-binomial Theorem (3.8) twice to the left side of (3.9), substitute the result in (3.5) then equate the coefficients of like powers of $t$.

We now determine the asymptotic behavior of $U_{n}(x ; a, b)$.

THEOREM 3.2. For fixed $x$ and $n \rightarrow \infty$ we have

$$
U_{n}(x ; a, b) \approx x^{n}(-a ; q)_{\infty} F\left(\frac{b}{x^{2}} ; a\right),
$$

where

$$
F(x ; a)=\sum_{0}^{\infty} \frac{(-1)^{k} x^{k}}{(q ; q)_{k}(-a ; q)_{k}} q^{k(k-1)} .
$$

Proof. Let $N \rightarrow \infty$ in (3.7). The interchanging of the summation and limit processes can be justified as in the Lommel polynomial case, see Watson $[14$, p. 305].

Our next result says something about the nature of the corresponding distribution function.

THEOREM 3.3. The polynomials $\left\{U_{n}(x ; a, b)\right\}$ satisfy the orthogonality relation

$$
\int_{-\infty}^{\infty} U_{n}(x ; a, b) U_{m}(x ; a, b) d \mu(x)=\frac{b^{n}(1+a)}{1+a q^{n}} q^{n(n-1) / 2} \delta_{m, n},
$$

where $d \mu$ is a purely discrete positive measure with bounded support and is normalized by $\int_{-\infty}^{\infty} d \mu(t)=1$. The only limit point of the support of $d \mu$ is $x=0$.

Proof. The corresponding monic polynomials are

$$
P_{n}(x):=\left\{(-a ; q)_{n}\right\}^{-1} U_{n}(x ; a, b) .
$$

They satisfy the three term recurrence relation

$$
P_{n}(x)=x p_{n-1}(x)-\lambda_{n} p_{n-1}(x), \quad n>0,
$$

with $P_{0}(x)=1, P_{-1}(x)=0$ and $\lambda_{n+1}=b q^{n-1}\left\{\left(1+a q^{n}\right)\left(1+a q^{n-1}\right)\right\}^{-1}$. The $c_{n}$ 's in Chihara's notation [4, Theorem 4.4, pp. 21-22] are all zero, and $\lambda_{n} \rightarrow 0$ as $n \rightarrow \infty$. The orthogonality relation (3.12) now follows from (1.2), p. 107 in [4] and the rest of the theorem follows from Theorem 3.5, p. 117 in [4], see also the discussion in the beginning of $\$ 3$, p. 113 in [4].

We now identify the Stieltjes transform of $d \mu$. 
THEOREM 3.4. The Stieltjes transform of $d \mu$ is given by

$$
\int_{-\infty}^{\infty} \frac{d \mu(t)}{z-t}=z^{-1} F\left(q b z^{-2} ; q a\right) / F\left(b z^{-2} ; a\right), \quad z \notin \operatorname{supp}\{d \mu\}
$$

where $F(z ; a)$ is defined by (3.11).

Proof. This follows from Markoff's theorem, Askey and Ismail [2], Pollaczek [9], Szegö [13, p. 57], since the left side of (3.11) is

$$
\lim _{n \rightarrow n} U_{n}^{*}(z ; a, b) / U_{n}(z ; a, b),
$$

which agrees with the right side upon using (3.10) and (3.4). This completes the proof.

It might be worth identifying the $U_{n}$ 's as basic hypergeometric polynomials. One can use (3.7) to prove the representations

$$
\begin{aligned}
& \text { (3.14) } U_{2 n}\left(x^{1 / 2} ; a, b\right) \\
& =q^{n^{2}-n}(-b)^{n}{ }_{r} \phi_{3}\left(\begin{array}{l}
q^{-n},-q^{1-n} / a,-a q^{n}, q^{n+1} ; q, q \frac{x a}{b} \\
-q, q^{1 / 2},-q^{1 / 2}
\end{array}\right) \\
& \begin{aligned}
&(3.15) \quad x^{-/ 2} U_{2 n+1}\left(x^{1 / 2} ; a, b\right) \\
&= q^{n^{2}-n} \frac{(-b)^{n}\left(1+a q^{n}\right)\left(1-q^{n+1}\right)}{(1-q)} \\
& \cdot{ }_{4} \phi_{3}\left(\begin{array}{l}
q^{-n},-q^{1-n} / a,-a q^{n+1}, q^{n+2} \\
-q, q^{3 / 2},-q^{3 / 2}
\end{array}, q \frac{x a}{b}\right) .
\end{aligned}
\end{aligned}
$$

4. The measure $d \mu(t)$. Before we can derive more precise information about the measure $d \mu(t)$ we shall establish three lemmas needed in the subsequence analysis.

LEMMA 4.1. The transcendental functions $F(z ; a)$ satisfy the $q$ ( geometric) difference equation

$$
(q z-a) F\left(z q^{2} ; a\right)+(a-q) F(z q ; a)+q F(z ; a)=0
$$

and the recurrence relation

$$
(1+a) q\{F(z ; a)-F(z / q ; a)\}=z F(z q ; a q) .
$$

Proof. Use (3.9) and straightforward manipulations. 
LEMMA 4.2. The functions $F(z ; a)$ and $F(q z ; q a)$ have no common zeros provided that $a \neq-q^{k}, k=0,1, \ldots$

Proof. If $\xi$ were a common zero of $F(z ; a)$ and $F(q z ; a q)$ then (4.2) would imply that $\xi$ is also a zero of $F(z / q ; a)$ which, by (4.1) would make $F(q \xi ; a)$ also vanish. We now use $(4.1)$ to deduce that $F\left(\xi q^{n} ; a\right)=0$, $n=1,2, \ldots$. This contradicts the identity theorem for analytic functions because $F(z ; a)$ is an entire transcendental function. This completes the proof.

LEMMA 4.3. All the zeros of $F(z ; a)$ are positive and simple when $a>-1$. There are infinitely many of them.

Proof. We saw in Theorem 3.3. that $\mu$ in (3.13) is a step function. Let $t_{1}, t_{2}, \ldots$ be the positive points of increase of $\mu(t)$ and let $\mu(t)$ have jump $A_{j}$ at $t=t_{j}>0, j=1,2, \ldots$. The polynomials $U_{n}(x ; a, b)$ are symmetric hence $\mu(t)$ must have a jump $A_{j}$ at $t=-t_{j}, j=1,2, \ldots$. The left side of (3.13) reduces to

$$
\int_{-\infty}^{\infty} \frac{d \mu(t)}{z-t}=\frac{A}{z}+\sum_{1}^{\infty}\left\{\frac{1}{z-t_{j}}+\frac{1}{z+t_{j}}\right\} A_{j}=\frac{A}{z}+\sum_{1}^{\infty} \frac{2 z A_{j}}{z^{2}-t_{j}^{2}},
$$

where $A$ is the mass at $t=0$. This establishes the Mittag-Leffler expansion

$$
\frac{A}{z}+\sum_{1}^{\infty} \frac{2 z A_{j}}{z^{2}-t_{j}^{2}}=z^{-1} F\left(q b z^{-2} ; q a\right) / F\left(b z^{-2} ; a\right) .
$$

Since $F\left(q b z^{-2} ; q a\right)$ and $F\left(b z^{-2} ; a\right)$ are entire transcendental functions with no common zeros, Lemmas 4.1 and 4.2 , we deduce from (4.3) that all the zeros of $F(b z ; a)$ must be of the form $t_{j}^{-2}$. Finally we prove that $A$ must be zero. From the theory of moments [11, pp. 42-46] we know that the jump at $t=0$ is $1 / \sum_{0}^{\infty} w_{n}^{2}(0),\left\{w_{n}(z)\right\}$ being the orthonormal polynomials. Now (3.12) and (3.1) imply

$$
w_{n}^{2}(z)=\frac{\left(1+a q^{n}\right) b^{-n}}{1+a} q^{-n(n-1) / 2} U_{n}^{2}(x ; a, b),
$$

and

$$
U_{2 n+1}(0 ; a, b)=0, \quad U_{2 n}(0 ; a, b)=(-b)^{n} q^{n(n-1)} .
$$

Hence $w_{2 n}^{2}(0) \sim q^{-n} /(1+a)$ and $\Sigma_{0}^{\infty} w_{n}^{2}(0)=\infty$. This shows that $A$, the jump at zero, must vanish and the proof is complete.

Let $\left\{z_{j}(a)\right\}$ be the zeros of $F(z ; a), a>-1$, ordered by

$$
0<z_{1}(a)<z_{2}(z)<\cdots<z_{n}(a)<z_{n+1}(a)<\cdots \text {. }
$$


THEOREM 4.4. When $a>-1$ we have

(i) The only cluster point of the sequence $\left\{z_{n}(a)\right\}$ is $+\infty$.

(ii) $F(q z ; q a)$ has an odd number of zeros in $\left(z_{n}(a), z_{n+1}(a)\right)$.

Proof. The conclusion (i) follows from the fact that $x=0$ is the only limit point of the support of $d \mu(t)$, see the Theorem 3.3. Now recall that the $A$ in (4.3) is zero. Rewrite the Mittag-Leffler expansion (4.3) in the form

$$
2 \sum_{1}^{\infty} \frac{b A_{j} t_{j}^{-2}}{b t_{j}^{-2}-z}=F(q z ; q a) / F(z ; a),
$$

hence $z_{j}(a)=b t_{j}^{-2}$ and

$$
2 b A_{j} z_{j}(z)=-F(q z(a) ; q a) F^{\prime}\left(z_{j}(a) ; a\right) .
$$

The sign of $F^{\prime}(z(a) ; a)$ is $(-1)^{j}$ because the $z_{j}$ 's are real and simple zeros. The left side of (4.5) is positive, thus the sign of $F\left(q z_{j}(a)\right.$; qa) is $(-1)^{j+1}$. This proves the assertion (ii).

COROLlaRY 4.5. The step function $\mu(t)$ has the jumps

$$
A_{j}=-\frac{F\left(q z_{j}(a) ; q a\right)}{F^{\prime}\left(z_{j}(a) ; a\right)}\left\{2 z_{j}(a)\right\}^{-1}
$$

at

$$
t_{j}= \pm \sqrt{b / z_{j}(a)} .
$$

In the rest of the present section we shall discuss the case $a=q$. This seems to be the only case where the zeros $\left\{z_{j}(a)\right\}$ can be computed explicitly. Clearly

$$
F(z ; q)=\sum_{0}^{\infty} \frac{(-z)^{n} q^{n(n-1)}}{\left(q^{2} ; q^{2}\right)}=\left(z ; q^{2}\right)_{\infty}
$$

follows from Euler's theorem

$$
\sum_{0}^{\infty} \frac{(-z)^{n} q^{n(n-1) / 2}}{(q ; q)_{n}}=(z ; q)_{\infty},
$$

Slater [11, p. 93]. The recursion (4.2) and (4.8) yield

$$
F\left(q z ; q^{2}\right)=\frac{(1+a) q}{z}\left\{\left(z ; q^{2}\right)_{\infty}-\left(z / q ; q^{2}\right)_{\infty}\right\} .
$$


The relationships (4.4), (4.6), (4.7) and (4.9) imply

(4.10) $z_{j}(q)=q^{-2 j}, \quad t_{j}= \pm \sqrt{b} q^{j}, \quad A_{j}=\frac{q^{j}\left(q^{3} ; q^{2}\right)_{j}\left(q ; q^{2}\right)_{\infty}}{2\left(q^{2} ; q^{2}\right)_{j}\left(q^{4} ; q^{2}\right)_{\infty}}$.

In this case the basic hypergeometric representations (3.14) and (3.15) reduce to

$$
U_{2 n}\left(x^{1 / 2}, q, b\right)=q^{n^{2}-n}(-b)^{n}{ }_{2} \phi_{1}\left(\begin{array}{c}
q^{-2 n}, q^{2 n+2} ; q^{2}, \frac{q^{2} x}{b} \\
q
\end{array}\right)
$$

and

$$
\begin{aligned}
& x^{-1 / 2} U_{2 n+1}\left(x^{1 / 2}, q, b\right) \\
& =q^{n^{2}-n}(-b)^{n} \frac{\left(1-q^{2 n+2}\right)}{1-q}{ }_{2} \phi_{1}\left(\begin{array}{c}
q^{-2 n}, q^{2 n+4} ; q^{2} ; \frac{q^{2} x}{b} \\
q^{3 / 2}
\end{array}\right) .
\end{aligned}
$$

These are the odd and even $q$-Jacobi polynomials and their orthogonality follows from the orthogonality relation

$$
\begin{gathered}
\sum_{j=0}^{\infty} \frac{\alpha^{j} q^{j}(\beta q ; q)_{j}}{(q ; q)_{j}} \phi_{n}^{\alpha, \beta}\left(q^{j}\right) \phi_{m}^{\alpha, \beta}\left(q^{j}\right) \\
=\frac{(q ; q)_{n}(\beta q ; q)_{n}\left(\alpha \beta q^{n+1} ; q\right)_{\infty} q^{n} \alpha^{n}}{(\alpha q ; q)_{n}(\alpha q ; q)_{\infty}\left(1-\alpha \beta q^{2 n+1}\right)} \delta_{m, n}
\end{gathered}
$$

for the $q$-Jacobi polynomials

$$
\phi_{n}^{\alpha, \beta}(x)={ }_{2} \phi_{1}\left(\begin{array}{c}
q^{-n}, \alpha \beta q^{n+1} \\
\alpha q
\end{array} ;, q x\right) .
$$

5. Continued fractions and generalizations. Recall that the asymptotic behaviour of $U_{n}(x ; a, b)$ was determined from the explicit formula (3.7). It can also be determined from the generating function (3.5) by applying Darboux' method, Olver [8, §8.9.2]. A comparison function is

$$
(1-x t)^{-1} \sum_{0}^{\infty} \frac{\left(\frac{b}{a} x^{-2} ; q\right)_{n}}{(q ; q)_{n}} a^{n} q^{n(n-1) / 2}
$$

This implies

$$
U_{n}(x ; a, b) \sim x^{n} \sum_{0}^{\infty} \frac{\left(\frac{b}{a} x^{-2} ; q\right)_{n}}{(q ; q)_{n}} a^{n} q^{n(n-1) / 2} .
$$


Upon comparing the asymptotic formulas (3.10) and (5.1) we obtain the identity

$$
\sum_{0}^{\infty} \frac{(b / a ; q)_{n}}{(q ; q)_{n}} a^{n} q^{n(n-1) / 2}=(-a ; q)_{\infty} \sum_{0}^{\infty} \frac{(-b)^{n}}{(q ; q)_{n}(-a ; q)_{n}} q^{n(n-1)}
$$

The identity (5.2) is known, but it is interesting that it follows from the above asymptotic analysis.

The continued fraction associated with the polynomials $\left\{U_{n}(z ; a, b)\right\}$ is

$$
\frac{1+a}{\sqrt{(1+a q) z}}-\frac{b}{\sqrt{\left(1+a q^{2}\right) z}}-\frac{b q}{\sqrt{\left(1+a q^{3}\right) z}}-\cdots
$$

The above continued fraction is also equal to

$$
\lim _{n \rightarrow \infty} U_{n}^{*}(z ; a, b) / U_{n}(a ; a, b),
$$

when the limit exists because $U_{n}$ and $U_{n}^{*}$ are the corresponding denominator and numerator polynomials, respectively. Note that the aforementioned limit can be evaluated via (3.10). Therefore the relationship

$$
\begin{gathered}
\frac{b+a}{\sqrt{(1+a q) z}}-\frac{b q}{\sqrt{\left(1+a q^{2}\right) z}}-\frac{1}{\sqrt{\left(1+a q^{3}\right) z}}-\cdots \\
=z^{-1} F\left(q b z^{-2} ; q a\right) / F\left(b z^{-2} ; a\right),
\end{gathered}
$$

holds when $b \neq 0$ and $b z^{-2}$ is not a zero of $F(z ; a)$. When $a>-1, b>0$, the continued fraction (5.3) converges if and only if $z \neq 0, z \neq$ $\pm \sqrt{b / j(a)}$. The case $a=1$ and $z=1$ gives the Ramanujan continued fraction

$$
\begin{aligned}
& \frac{1}{\prod_{1}}+\frac{b q \mid}{\lceil 1}+\frac{b q^{2}}{\lceil 1}+\cdots \\
& =\left\{\sum_{0}^{\infty} b^{k} q^{k^{2}} /(q ; q)_{k}\right\}\left\{\sum_{0}^{\infty} b^{n} q^{n^{2}+n} /(q ; q)_{n}\right\}^{-1},
\end{aligned}
$$

see Andrews [1, p. 104]. There is no loss of generality in setting $z=1$ in (5.3), as can be seen by replacing $b$ by $b z^{-2}$ and consider the continued fraction whose $n$th convergent is

$$
z^{-n+1} U_{n}^{*}(z ; a, b) / z^{-n} U_{n}(z ; a, b) .
$$

The continued fraction (5.3), with $z=1$, was stated in Ramanujan's Notebooks [10, p. 196]. Upon applying (5.2) to (5.3), with $z=1$, we obtain a result of Carlitz [3]. 
Hirschhorn [6] considered the continued fraction as a function of $\beta$

$$
\frac{1}{1+\alpha+\beta}+\frac{\gamma q-\alpha}{\sqrt{1+\alpha+\beta q}}+\frac{\gamma q^{2}-\alpha}{\sqrt{1+\alpha+\beta q^{2}}}+\cdots .
$$

The denominator polynomials are more general than our $U_{n}$ 's as functions of the variable $a$ for fixed $z$. The support of the corresponding measure is unbounded. The orthogonality relation for the Hirschhorn polynomials is not known. The associated moment problem is indeterminate as can be seen from Carleman's criterion, see [11, p. 59].

We now consider the most general symmetric solution to the recursion (3.1). Let

$$
p_{n+1}(x ; a, b)=x\left(1+a q^{n}\right) p_{n}(x ; a, b)-b q^{n-1} p_{n-1}(x ; a, b) .
$$

There is no loss of generality in letting

$$
p_{0}(x)=1, \quad p_{1}(x)=c x .
$$

Clearly $p_{n}(x ; a, b)$ reduces to $U_{n}(x ; a, b)$ when $c=a+\cdot 1$. The recurrence relation (5.6) has the fundamental system $\left\{U_{n}(x ; a, b), U_{n}^{*}(x ; a, b)\right\}$. Hence

$$
p_{n}(x ; a, b)=U_{n}(x ; a, b)+\frac{x(c-a-1)}{1+a} U_{n}^{*}(x ; a, b), \quad n>0,
$$

and

$$
p_{n}^{*}(x ; a, b)=\frac{c}{1+a} U_{n}^{*}(x ; a, b) .
$$

The proofs of the following results are similar to the proofs of the corresponding results when $c=1$ and will be omitted.

THEOREM 5.1 (Orthogonality Relation). When $c>0, b>0,1+a q>0$, we have

$$
\begin{aligned}
\int_{-\infty}^{\infty} p_{n}(x ; a, b) p_{m}(x ; a, b) d \psi(x) \\
=\frac{b^{n} c q^{n(n-1) / 2}}{1+a q^{n}} \delta_{m, n}, \quad m, n>0
\end{aligned}
$$

where $d \mu$ is a purely discrete positive measure with bounded support and normalized by $\int_{-\infty}^{\infty} d \psi=1$. The origin is the only limit point of the support of $d \psi$. 
THEOREM 5.2. The Stieltjes transform of $d \psi$ is

$$
\begin{aligned}
\int_{-\infty}^{\infty} \frac{d \psi(t)}{z-t}=\frac{c}{z} F\left(b q z^{-2} ; q a\right)\{ & (1+a) F\left(b z^{-2} ; a\right) \\
& \left.+(c-a-1) F\left(b q z^{-2} ; q a\right)\right\}^{-1},
\end{aligned}
$$

for $z \notin \operatorname{supp}\{d \psi\} a>-1, c>0$, where $F(z ; a)$ is as in (3.11).

THEOREM 5.3. When a $q>-1, c>0$, the transcendental function

$$
\phi(z):=(1+a) F(z ; a)+(c-a-1) F(q z ; q a)
$$

has infinitely many zeros. The zeros are positive and simple. Furthermore $F(q z ; q a)$ vanishes an odd number of times between two sucessive zeros of $\phi(z)$. The only cluster point of zeros of $\phi(z)$ is $\pm \infty$.

Note that Theorem 5.3 reduces to Lemma 4.3 and Theorem 4.4 when $c=a+1$.

Acknowledgment. The authors gratefully acknowledge Richard Askey's critical reading of an earlier version of this work. His kind suggestions improved the content and presentation of this work. Thanks to Diane Mowers for her excellent typing.

\section{REFERENCES}

1. G. E. Andrews, The Theory of Partitions, Addison-Wesley, Reading, Mass., 1976.

2. R. Askey and M. E. H. Ismail, Recurrence relations, continued fractions and orthogonal polynomials, to appear.

3. L. Carlitz, Note on some continued fractions of the Rogers-Ramaujan type, Duke Math. J., 32 (1965), 713-720.

4. T. Chihara, An Introduction to Orthogonal Polynomials, Gordon and Breach, New York, 1978.

5. J. Geronimus, On a set of polynomials, Ann. Math., (2) 31 (1930), 681-686.

6. M. Hirschhorn, Developments in the theory of partitions, Doctoral Dissertation, University of New South Wales, 1979.

7. M. E. H. Ismail, The zeros of basic Bessel functions, the functions $J_{y+a x}(x)$ and associated orthogonal polynomials, J. Math. Anal. Appl., 86 (1982), 1-19.

8. F. W. J. Olver, Asymptotics and Special Functions, Academic Press, New York, 1974.

9. F. Pollaczek, Sur une generalisation des polynômes de Jacobi, Memorial des Sciences Mathematique, volume 121, Paris, 1956.

10. S. Ramanujan, Notebooks, Tata Institute, Bombay, 1957.

11. J. A. Shohat and J. D. Tamarkin, The Problem of Moments, Mathematical Surveys, volume I, American Mathematical Society, Providence, R.I., 1963.

12. L. J. Slater, Generalized Hypergeometric Functions, Cambridge University Press, Cambridge, 1966.

13. G. Szegö, Orthogonal Polynomials, Fourth edition, Colloquium Publications, volume 23, American Mathematical Society, Providence, R.I., 1975. 
14. G. N. Watson, A Treatise on the Theory of Bessel Functions, second edition, Cambridge University Press, Cambridge, 1944.

Received April 21, 1981. First author's research partially supported by the Natural Science and Engineering Research Council of Canada. Second author's research partially supported by NSF Grant MCS-8002539.

UNIVERSITY OF AlbERTA, EDMONTON

Alberta, Canada T6G 2G1

AND

Arizona State University

TEMPE, AZ 85281 



\title{
PACIFIC JOURNAL OF MATHEMATICS EDITORS
}

\author{
Donald BABBITT (Managing Editor) \\ University of California \\ Los Angeles, CA 90024 \\ Hugo Rossi \\ University of Utah \\ Salt Lake City, UT 84112 \\ C. C. Moore and Arthur Ogus \\ University of California \\ Berkeley, CA 94720
}

\author{
J. Dugundi \\ Department of Mathematics \\ University of Southern California \\ Los Angeles, CA 90089-1113
}

R. FinN and H. SAMELSON

Stanford University

Stanford, CA 94305

\section{ASSOCIATE EDITORS}
R. ARENS
E. F. BECKENBACH
B. H. NeumanN
F. WOLF
K. YoSHIDA (1906-1982)

\section{SUPPORTING INSTITUTIONS}

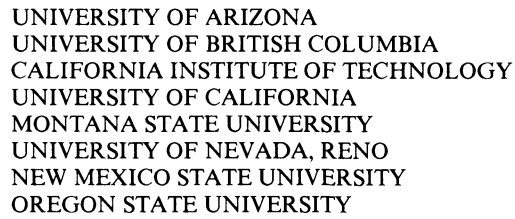

UNIVERSITY OF ARIZONA

UNIVERSITY OF BRITISH COLUMBIA

CALIFORNIA INSTITUTE OF TECHNOLOGY

UNIVERSITY OF CALIFORNIA

MONTANA STATE UNIVERSITY

UNIVERSITY OF NEVADA, RENO

NEW MEXICO STATE UNIVERSITY

OREGON STATE UNIVERSITY

\author{
UNIVERSITY OF OREGON \\ UNIVERSITY OF SOUTHERN CALIFORNIA \\ STANFORD UNIVERSITY \\ UNIVERSITY OF HAWAII \\ UNIVERSITY OF TOKYO \\ UNIVERSITY OF UTAH \\ WASHINGTON STATE UNIVERSITY \\ UNIVERSITY OF WASHINGTON
}

The Supporting Institutions listed above contribute to the cost of publication of this Journal, but they are not owners or publishers and have no responsibility for its content or policies.

Mathematical papers intended for publication in the Pacific Journal of Mathematics should be in typed form or offset-reproduced (not dittoed), double spaced with large margins. Please do not use built up fractions in the text of the manuscript. However, you may use them in the displayed equations. Underline Greek letters in red, German in green, and script in blue. The first paragraph must be capable of being used separately as a synopsis of the entire paper. In particular it should contain no bibliographic references. Please propose a heading for the odd numbered pages of less than 35 characters. Manuscripts, in triplicate, may be sent to any one of the editors. Please classify according to the scheme of Math. Reviews, Index to Vol. 39. Supply name and address of author to whom proofs should be sent. All other communications should be addressed to the managing editor, or Elaine Barth, University of California, Los Angeles, California 90024.

There are page-charges associated with articles appearing in the Pacific Journal of Mathematics. These charges are expected to be paid by the author's University, Government Agency or Company. If the author or authors do not have access to such Institutional support these charges are waived. Single authors will receive 50 free reprints; joint authors will receive a total of 100 free reprints. Additional copies may be obtained at cost in multiples of 50 .

The Pacific Journal of Mathematics is issued monthly as of January 1966. Regular subscription rate: $\$ 132.00$ a year (6 Vol., 12 issues). Special rate: $\$ 66.00$ a year to individual members of supporting institutions.

Subscriptions, orders for numbers issued in the last three calendar years, and changes of address should be sent to Pacific Journal of Mathematics, P.O. Box 969, Carmel Valley, CA 93924, U.S.A. Old back numbers obtainable from Kraus Periodicals Co., Route 100, Millwood, NY 10546.

The Pacific Journal of Mathematics ISSN 0030-8730 is published monthly by the Pacific Journal of Mathematics at P.O. Box 969, Carmel Valley, CA 93924. Application to mail at Second-class postage rates is pending at Carmel Valley, California, and additional mailing offices. Postmaster: Send address changes to Pacific Journal of Mathematics, P. O. Box 969, Carmel Valley, CA 93924.

PUBLISHED BY PACIFIC JOURNAL OF MATHEMATICS, A NON-PROFIT CORPORATION

Copyright $\odot 1983$ by Pacific Journal of Mathematics 


\section{Pacific Journal of Mathematics}

Vol. 104, No. $2 \quad$ June, 1983

Leo James Alex, Simple groups and a Diophantine equation ........... 257

Herbert James Alexander and John Wermer, On the approximation of

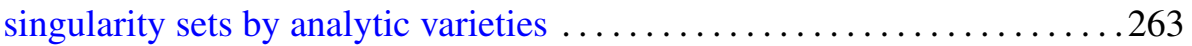

Waleed A. Al-Salam and Mourad Ismail, Orthogonal polynomials associated with the Rogers-Ramanujan continued fraction .......... 269

J. L. Brenner and Roger Conant Lyndon, Permutations and cubic

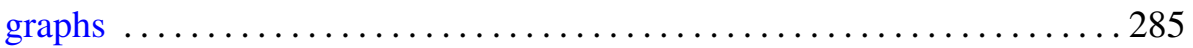

Ian George Craw and Susan Ross, Separable algebras over a commutative

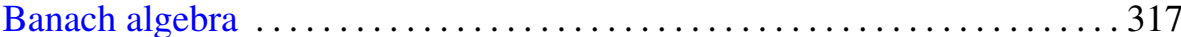

Jesus M. Dominguez, Non-Archimedean Gel'fand theory ............. 337

David Downing and Barry Turett, Some properties of the characteristic of

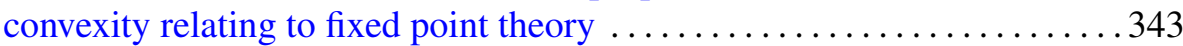

James Arthur Gerhard and Mario Petrich, Word problems for free objects in certain varieties of completely regular semigroups $\ldots \ldots \ldots 351$

Moses Glasner and Mitsuru Nakai, Surjective extension of the reduction

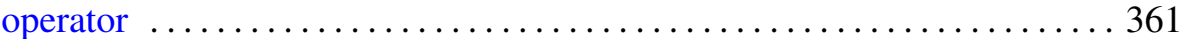

Takesi Isiwata, Ultrafilters and mappings $\ldots \ldots \ldots \ldots \ldots \ldots \ldots \ldots \ldots \ldots \ldots$

Lowell Duane Loveland, Double tangent ball embeddings of curves in $E^{3}$

Douglas C. McMahon and Ta-Sun Wu, Homomorphisms of minimal flows

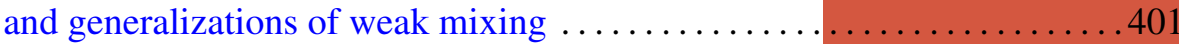

P. H. Maserick, Applications of differentiation of $\mathscr{L}_{p}$-functions to semilattices

Wayne Bruce Powell and Constantine Tsinakis, Free products in the class of abelian $l$-groups

Bruce Reznick, Some inequalities for products of power sums

C. Ray Rosentrater, Compact operators and derivations induced by

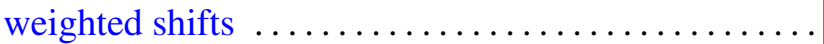

Edward Silverman, Basic calculus of variations

Charles Andrew Swanson, Criteria for oscillatory sublinear Schrödinger equations

David J. Winter, The Jacobson descent theorem 